\title{
Immune response pathways enriched in breast cancer samples with brain metastasis
}

\author{
Peng Wang ${ }^{1,2,3,4}$, Zengfeng Sun ${ }^{1,2,3,4}$, Zhen Zhang ${ }^{1,2,3,4}$, Qiang Yin Yi,3,4 $^{1,3}$ \\ ${ }^{1}$ Department of Neuro-Oncology and Neurosurgery, Tianjin Medical University Cancer Institute and Hospital, Tianjin, China; ${ }^{2}$ National Clinical \\ Research Center for Cancer, Tianjin, China; ${ }^{3}$ Key Laboratory of Cancer Prevention and Therapy, Tianjin, China; ${ }^{4}$ Tianjin's Clinical Research Center \\ for Cancer, Tianjin, China \\ Contributions: (I) Conception and design: P Wang, Q Yin; (II) Administrative support: P Wang; (III) Provision of study materials or patients: P Wang; \\ (IV) Collection and assembly of data: P Wang, Q Yin, Z Sun; (V) Data analysis and interpretation: P Wang, Z Zhang; (VI) Manuscript writing: All \\ authors; (VII) Final approval of manuscript: All authors. \\ Correspondence to: Peng Wang, MD, PhD. Department of Neuro-Oncology and Neurosurgery, Tianjin Medical University Cancer Institute \\ and Hospital, National Clinical Research Center for Cancer, Tianjin's Clinical Research Center for Cancer, Tianjin 300060, China. \\ Email: pengwang@tmu.edu.cn.
}

Backgroundk Breast cancer (BC) is the most common form of cancer in women. BC brain metastasis (BM) is associated with poor prognosis, especially for Triple negative breast cancer (TNBC). However, the driver genes of this clinical characteristic are poorly understood.

Methods: This study conducted a transcriptome-wide analysis of gene expression levels in BCBM samples from the Gene Expression Omnibus (GEO) and The Cancer Genome Atlas (TCGA) datasets. Clinical data and gene expression matrix of TNBC samples were collected. Differential analysis and functional enrichment of metastasis vs. non metastasis data samples were conducted. Genes associated with overall survival and BM event was scanned.

Results: Up-regulation in 120 genes and down-regulation in 56 genes were found in TNBC metastasis data. Gene Ontology (GO) and Kyoto Encyclopedia of Genes and Genomes (KEGG) clustering using expression alternated genes showed unique immune-gene enrichment in BM samples. Immune response category GO:000695 was found as the most significant term associated with metastasis event. KEGG pathways including cytokine pathways and Primary immunodeficiency were significantly changed in metastasis samples. ESR1 and FYB2 genes expression changes were found to be linked to survival or BM events.

Conclusions: Our results suggest that data-mining on the immune microenvironment of BM might be useful in future study.

Keywords: Breast cancer (BC); brain metastasis (BM); transcriptome; differential expression analysis; immune profile

Submitted Oct 15, 2021. Accepted for publication Dec 08, 2021.

doi: $10.21037 / g s-21-745$

View this article at: https://dx.doi.org/10.21037/gs-21-745

\section{Introduction}

Breast cancer (BC) is the most common form of cancer in women, accounting for almost one-third of cancer cases among US women (1). With considerable increases in diagnosis rates, mostly due to better imaging and screening, the rate of cancer deaths has steadily declined since 1975. BC brain metastasis (BM), which occurs 34 months (on average) after diagnosis of $\mathrm{BC}$, is a sign of poor prognosis, with a mean survival time of 15 months. A random sampling of $\mathrm{BC}$ patients shows that approximately $15 \%$ have diagnosed BM. Owing to better detection methods, there has been a trend of increasing BM rates over time, with BM being detected in up to $30 \%$ of all BC patients in autopsies. Human epidermal growth factor receptor 2 positive (HER2 ${ }^{+}$) BC patients have 
an up to $50 \%$ chance of being diagnosed with BM, while triple-negative patients have a $25-46 \%$ chance $(2,3)$.

The risk of $\mathrm{BCBM}$ is correlated with certain mutations. A gene panel from 2015 with a sample size of 60 has shown that mutations in TP53 (59.5\%), MLH1 (14.3\%), and PIK3CA (14.3\%) were relatively common, and that there were no significant genetic differences between BM and non-BM BC cells (4). A DNA panel of over 10,000 cancer patients from 2017 demonstrated similar mutation rates for TP53 and PIK3CA in unrelated cancer cells (5). More recent analyses have shown considerable genetic differences between primary BC cells and BM cells, with BM cells having more mutations and copy-number-alterations (6). A 2007 study of 44 BC cases with 23 BMs showed that while only $15-20 \%$ of $\mathrm{BC}$ in general are estrogen receptor negative $\left(\mathrm{ER}^{-}\right)$and progesterone receptor negative $\left(\mathrm{PR}^{-}\right)$, more than $64 \%$ of $\mathrm{BCBMs}$ are steroid hormone receptor negative (7). Triple negative breast cancer (TNBC) is defined as $\mathrm{BC}$ that is $\mathrm{ER}^{-}, \mathrm{PR}^{-}$, and $\mathrm{HER} 2^{-}$, and has a survival rate between those of steroid receptor-positive and HER2 ${ }^{+}$BC; although, it is the most likely to have recurrent metastases (8). The HER2 gene is closely related to HER1 (also known as epidermal growth factor receptor, $E G F R$ ) and is targeted for treatment in roughly $30 \%$ of BC patients (9). Both are known oncogenes and activate mechanisms for cell survival, proliferation, migration, and angiogenesis, all of which are factors that contribute to oncogenesis and metastasis (10). An analysis of 20 BCBM patient genomes has shown gain-of-function in FGFR4 (30\%) and loss of function in ESR1 (45\%). In addition, the expression of ERBB2/HER2 were found increased greater than two-fold in $35 \%$ of patients (11).

Overall, many effective treatments for $\mathrm{BC}$ are less effective for BCBM (12). A monoclonal-antibody-based treatment, trastuzumab, targets the HER2 receptor and is considered one of the most effective treatments for HER2 ${ }^{+}$ BC. However, it is less effective in BCBM compared to combination chemotherapy (13). Despite brain tumors secreting angiogenic factors, which result in a blood-tumor barrier (BTB) that is more porous than the blood-brain barrier (BBB), antibodies are still too large to effectively cross the BTB (14). In addition, the exact molecular mechanisms for BCBM are uncertain, although signal proteins like C-X-C Motif chemokine receptor 4 (CXCR4) and Vascular Endothelial Growth Factor (VEGF) are known to play a role (12). However, the use of trastuzumab and other antibody therapies prior to BM have been shown to decrease the probability of BM in cases of metastatic
HER2 ${ }^{+}$BC, from a 1-year BCBM probability of $87.7 \%$ for the control group to $66.9 \%$ for the test group (15). Interestingly, the resistance of $\mathrm{HER}^{+} \mathrm{BCBM}$ to $\mathrm{HER} 2^{+}$ targeted therapy seems to extend to some small-molecule treatments, although combination therapies have shown a significant effect $(16,17)$. A 2010 study reported considerable differences in BCBM patient survival times by type and treatment. For example, the mean survival time of TNBC $\mathrm{BM}$ patients was 4 months, $\mathrm{HER}^{+}$patients was 9 months, and luminal HER2 ${ }^{+}$patients was 15 months. HER2 ${ }^{+}$ BCBM had an untreated mean survival time of 3 months, which increased to 8 months with chemotherapy, and 11 months with chemotherapy + radiation (18). Recently, cell proliferation, immune response and cell migration as critical pathways in a prognostic signature for $\mathrm{HER} 2^{+}: \mathrm{ER} \alpha^{-}$breast cancer using Gene Set Enrichment Analysis (GSEA) (19). Cancer immunologic pathways were found related to clinical outcomes in basal-like breast cancer (20). Immune response pathways were speculated as potential novel drivers in brain metastatic samples of TNBC samples with homologous recombination (HR) signature conserved (21). Whether immune pathway affects TNBC brain metastases needs to be checked in transcriptome data. To test this assumption, this study conducted a transcriptome-wide analysis of TNBC BCBM. Our findings can help to improve the outcomes of TNBC BCBM patients. We present the following article in accordance with the MDAR reporting checklist (available at https://dx.doi.org/10.21037/gs-21-745).

\section{Methods}

\section{Data collection}

Messenger RNA (mRNA) expression data for 204 samples from the GSE12276 dataset (22) was downloaded from the Gene Expression Omnibus (GEO) database (https://www.ncbi.nlm.nih.gov/geo/) using R package GEOquery (23). This was the total BCBM expression available from the GEO database. The probe with the highest expression was used as the gene expression value for each gene. Probes failing to map and outlier samples were discarded. In total 15,222 genes in 198 samples were used in following analysis. The study was conducted in accordance with the Declaration of Helsinki (as revised in 2013).

\section{Bioinformatics analysis}

Differential analysis of gene expression between primary samples with/without metastasis was conducted using 


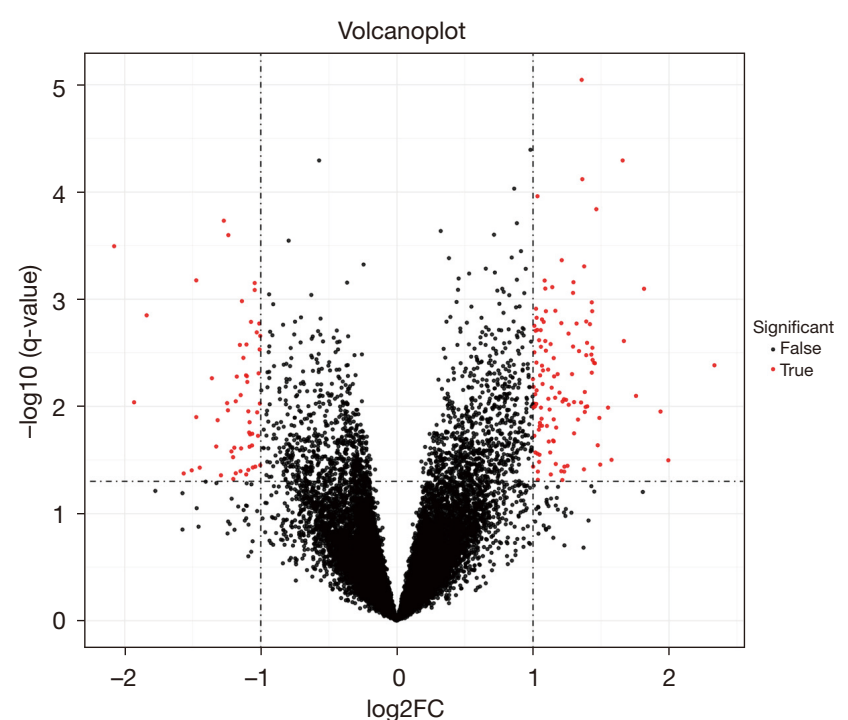

Figure 1 Volcano plot of differential gene expression. The vertical axis represents statistical significance while the horizontal axis represents the size of expression difference. The red dots represent genes that meet the significance thresholds for both.

R-package DESeq2 (24). Samples without metastasis were used as control group. Significant genes were selected using an adjusted $\mathrm{P}$ value cutoff of 0.05 and absolute value of $\log 2 \mathrm{FC}>1$. Functional enrichment was investigated using the R Clusterprofiler package (25). R-package ggplot2 (26) was used to visualize the top enriched terms of the Gene Ontology (GO) and Kyoto Encyclopedia of Genes and Genomes (KEGG) databases, using $\mathrm{P}<0.05$ as the significance threshold. The TNBC-related gene list and external verification gene list were extracted from The Cancer Genome Atlas Breast Invasive Carcinoma (TCGABRCA) using R-package GEOquery. The code for this paper is available upon request.

\section{Survival analysis}

In addition, $\mathrm{R}$ package survminer (27) was used to conduct 3 -year survival and $\mathrm{BM}$ analyses, using $\mathrm{P}$ value $<0.05$ as the significance threshold.

\section{Statistical analysis}

All statistical analysis were conducted in R 3.8.

\section{Results}

The GSE12276 dataset were used to conduct BCBM analysis. The expression levels were normalized and transformed through $\log 2+1$. The differential expression (DE) analysis results showed up-regulation in 120 genes and down-regulation in 56 genes (Figure 1) for BM in TNBC, as opposed to non-metastatic TNBC.

\section{GO enrichment}

GO enrichment analysis of the differentially-expressed genes showed that $97 \mathrm{DE}$ genes were classified as biological processes (BP), two as cellular components (CC), and 20 as molecular functions (MF) (Figure 2A). The most statistically significant and differentially expressed GO group was GO:000695, which was the group for immune response. A more detailed analysis of GO enrichment indicated that the enriched immune response contained categories with a humoral immune response, immune signaling, immune chemotaxis, immune chemokine, and calcium ion hemostasis (Figure 2B).

\section{KEGG enrichment}

KEGG enrichment results showed enrichment in multiple immune pathways (Figure 3). Notably, cytokines are involved with immune-cell-induced apoptosis, and dysfunction in cytokine pathways are associated with oncogenesis and proliferation (28). Primary immunodeficiency is similarly associated with cancer, and the interleukin-17 (IL-17) signaling pathway has been shown to directly cause BC through granulocyte colony-stimulating factor (29). Overall, KEGG analysis results showed a systematic enrichment of genes related to oncogenesis and cancer progression, with anti-apoptotic and immune system pathways increasing the survival of mutated cells and inflammation pathways, resulting in increased cancer proliferation and metastasis.

\section{Survival analysis}

Our survival analysis of gene expression identified estrogen receptor alpha (ESR1) as the only statistically significant gene (Table 1). ESR1 mutations are frequently found in metastatic BC, and are often acquired after aromatase inhibitor treatments for metastatic BC (30).

Metastasis analysis identified FYB2 as the only statistically significant gene, which is part of T-cell receptor-mediated signaling pathway activation (Table 2). Validation results showed similar trends, with no gene DE levels related to BM events. Furthermore, there was no 


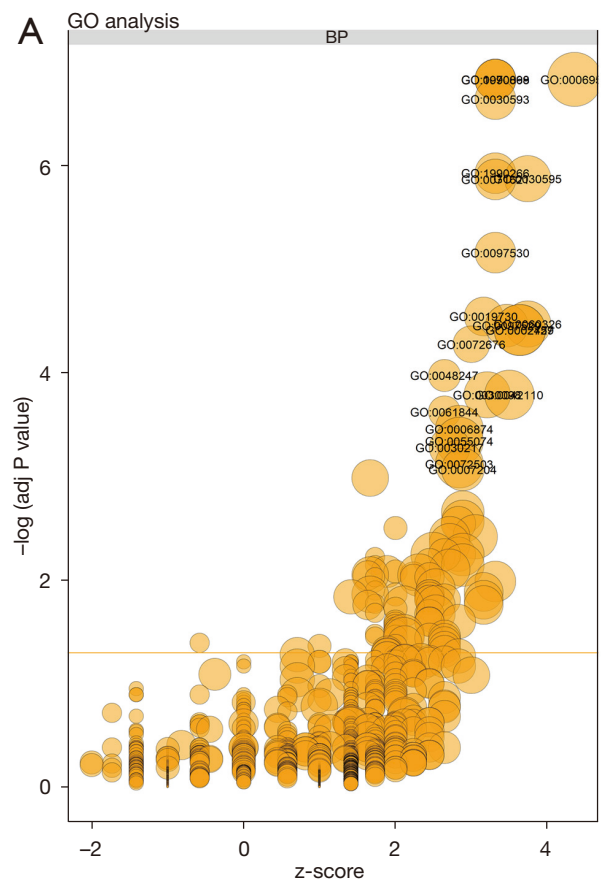

B

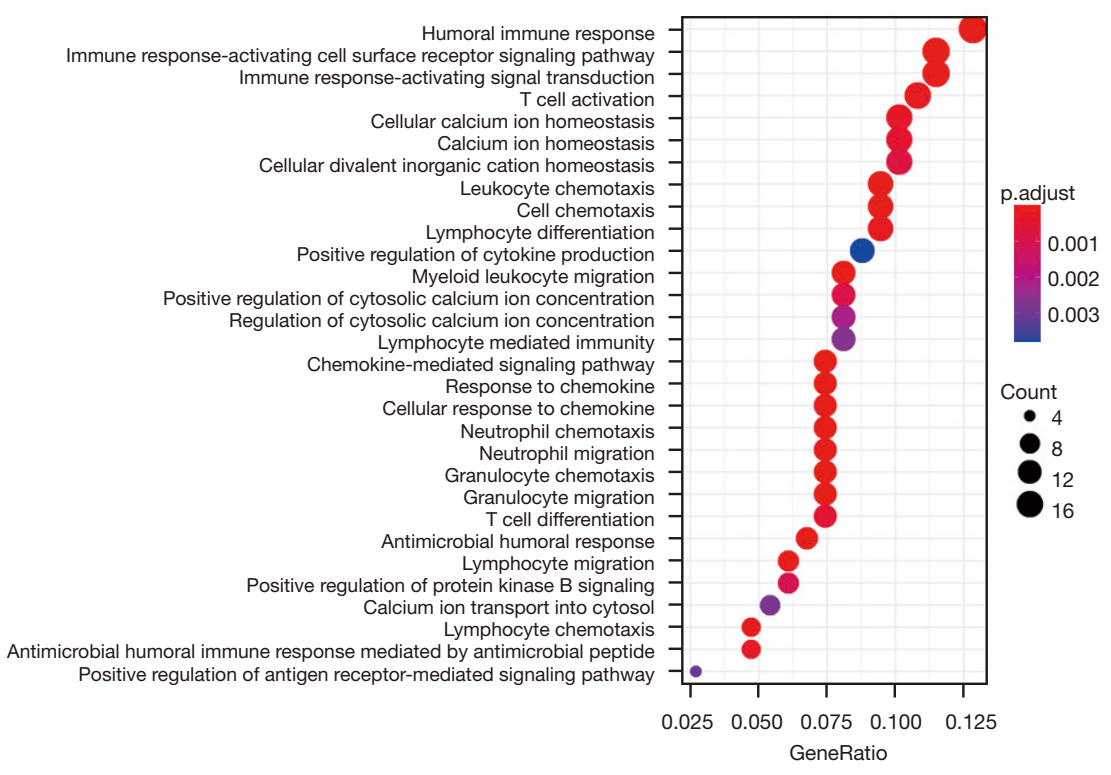

Figure 2 Bubble plot of Gene Ontology (GO) enrichment analysis (A) and detailed inspection of immune-related processes (B). (A) The horizontal axis represents size of effect and the vertical axis represents statistical significance. The circles represent GO groups, with size representing the number of differentially-expressed genes within a GO group. (B) The horizontal axis represents gene ratio. The dot size shows gene count, and the red color indicates smaller adjusted $\mathrm{P}$ values.

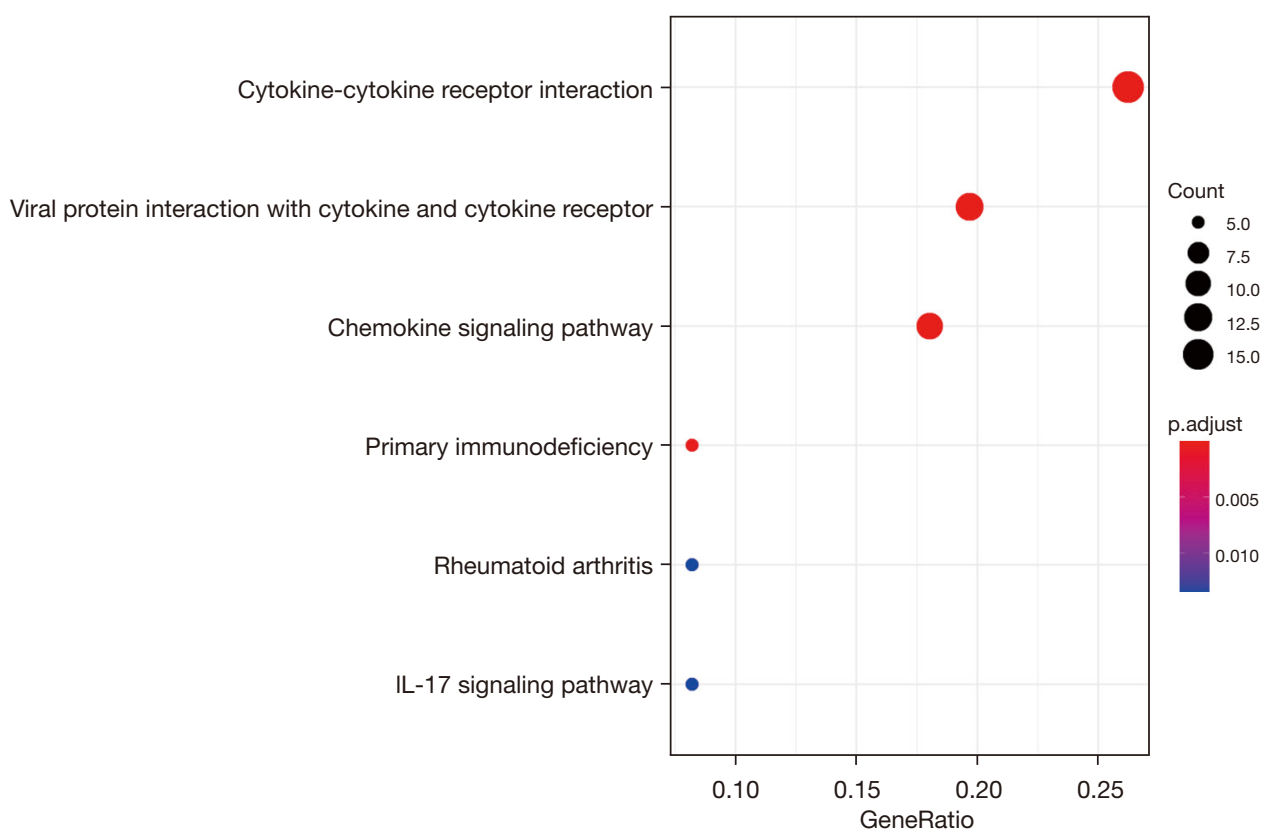

Figure 3 Kyoto Encyclopedia of Genes and Genomes (KEGG) pathway enrichment of differential expression (DE) genes. The horizontal axis represents gene ratioes of $\mathrm{DE}$ genes in pathways. The dot size shows gene count, and the red color shows the smaller adjusted $\mathrm{P}$ value. 
Table 1 Differentially expressed genes in 3-year survival

\begin{tabular}{lccc}
\hline Term & HR $(95 \%$ Cl $)$ & Statistics & P value \\
\hline ESR1 & $-0.21(-0.32$ to -0.09$)$ & -3.65 & $<.01$ \\
PRR15 & $-0.13(-0.27-0.02)$ & -1.73 & 0.08 \\
ERBB4 & $0.09(-0.01-0.2)$ & 1.72 & 0.09 \\
BCL11A & $-0.08(-0.22-0.05)$ & -1.27 & 0.2 \\
VGLL1 & $-0.04(-0.11-0.03)$ & -1.11 & 0.26 \\
MMP1 & $0.04(-0.03-0.1)$ & 1.09 & 0.27 \\
HORMAD1 & $-0.03(-0.11-0.04)$ & -0.85 & 0.4 \\
FYB2 & $-0.04(-0.14-0.05)$ & -0.84 & 0.4 \\
LINC00993 & $-0.03(-0.11-0.06)$ & -0.61 & 0.54 \\
CA12 & $-0.04(-0.18-0.11)$ & -0.52 & 0.6 \\
TSPAN1 & $-0.02(-0.12-0.09)$ & -0.35 & 0.73 \\
ANXA9 & $-0.01(-0.13-0.12)$ & -0.11 & 0.91 \\
\hline
\end{tabular}

Table 2 Differentially expressed genes in brain metastasis

\begin{tabular}{lccc}
\hline Term & Estimate & SE & Statistics \\
\hline Intercept & -8.48 & 6.09 & -1.39 \\
FYB2 & -0.53 & 0.19 & -2.73 \\
ERBB4 & -0.15 & 0.18 & -0.86 \\
CA12 & -0.04 & 0.26 & -0.16 \\
VGLL1 & 0 & 0.12 & -0.03 \\
ESR1 & 0.01 & 0.19 & 0.06 \\
TSPAN1 & 0.11 & 0.19 & 0.58 \\
LINC00993 & 0.09 & 0.16 & 0.59 \\
BCL11A & 0.18 & 0.25 & 0.71 \\
PRR15 & 0.26 & 0.26 & 1 \\
MMP1 & 0.21 & 0.15 & 1.44 \\
HORMAD1 & 0.19 & 0.13 & 1.49 \\
\hline
\end{tabular}

SE, standard error.

difference in primary and meta-samples in our dataset for TNBC-related genes.

\section{Discussion}

In this study, we attempted to identify the differential gene expression differences between TNBC and non-TNBC. Multiple genes were found to be associated with BCBM, with 120 up-regulated and 56 down-regulated genes. These genes were enriched in the following categories: humoral immune response, immune signaling, immune chemotaxis, immune chemokine, and calcium ion hemostasis. The humoral immune response directly attacks and helps to induce apoptosis in cancer cells, and has been shown to have predictive value for BC survival (31). Immune signaling is a vital aspect of inflammation pathways and contributes 
directly to cancer proliferation (32). Immune chemotaxis and immune chemokine are both functions that help guide immune cells to cancerous and pre-cancerous cells for destruction, and disruption helps cancer cells evade the immune system (33). High levels of cellular calcium are known to play a role in the calcification of breast tissue, which is associated with BC progression (34). Primary immunodeficiency is similarly associated with cancer, and the IL-17 signaling pathway has been shown to directly cause BC through the granulocyte colony-stimulating factor (29). Through multivariate analysis, we also found that ESR1 and $F Y B 2$ were independently linked to survival and metastasis. ESR1 is a well-known metastatic and drug-resistance gene in BC $(35,36)$. Recent real-word data and cell line studies have found infiltrating immune cell status reveals therapeutic vulnerabilities of ESR1-mutant breast cancer $(37,38)$. FYB2 is part of the T-cell receptor-mediated activation of signaling pathways and T-cell activation (39); however, its relationship to cancer has not been specifically investigated.

Compared to other forms of BC, TNBC has different survival and metastasis patterns, and is generally associated with lower survival and a higher likelihood of metastasis (8). There have been few reports regarding gene expression characteristics specific to metastatic TNBC (40). Much of this has been due to a limited number of samples available for comparative analysis (41). Our re-analysis found that FYB2 may act as a key differentially-expressed gene in TNBC BM. T-cell activation plays a vital role in regulating cancer proliferation and metastasis (42). FYB2 might provide a novel target of drug discovery.

In conclusion, this study confirmed that metastasis is closely correlated to the expression of inflammation- and immune-related genes. Notably, immunotherapy, which affects both, is known to decrease both the likelihood of further BMs and increase survival in patients with metastatic melanoma (43).

\section{Acknowledgments}

We are grateful to Dr. Yunjing Wang for helpful discussions and critical reading of our draft manuscript.

Funding: None.

\section{Footnote}

Reporting Checklist: The authors have completed the MDAR reporting checklist. Available at https://dx.doi. org/10.21037/gs-21-745
Conflicts of Interest: All authors have completed the ICMJE uniform disclosure form (available at https://dx.doi. org/10.21037/gs-21-745). The authors have no conflicts of interest to declare.

Etbical Statement: The authors are accountable for all aspects of the work in ensuring that questions related to the accuracy or integrity of any part of the work are appropriately investigated and resolved. The study was conducted in accordance with the Declaration of Helsinki (as revised in 2013).

Open Access Statement: This is an Open Access article distributed in accordance with the Creative Commons Attribution-NonCommercial-NoDerivs 4.0 International License (CC BY-NC-ND 4.0), which permits the noncommercial replication and distribution of the article with the strict proviso that no changes or edits are made and the original work is properly cited (including links to both the formal publication through the relevant DOI and the license). See: https://creativecommons.org/licenses/by-nc-nd/4.0/.

\section{References}

1. Floridi C, Cellina M, Buccimazza G, et al. Ultrasound imaging classifications of thyroid nodules for malignancy risk stratification and clinical management: state of the art. Gland Surg 2019;8:S233-44.

2. Shi YX, Chen L, Liu YC, et al. Differences among the Thyroid Imaging Reporting and Data System proposed by Korean, the American College of Radiology and the European Thyroid Association in the diagnostic performance of thyroid nodules. Transl Cancer Res 2020;9:4958-67.

3. Yim Y, Baek JH. Core needle biopsy in the management of thyroid nodules with an indeterminate fine-needle aspiration report. Gland Surg 2019;8:S77-85.

4. Lee JY, Park K, Lim SH, et al. Mutational profiling of brain metastasis from breast cancer: matched pair analysis of targeted sequencing between brain metastasis and primary breast cancer. Oncotarget 2015;6:43731-42.

5. Zehir A, Benayed R, Shah RH, et al. Mutational landscape of metastatic cancer revealed from prospective clinical sequencing of 10,000 patients. Nat Med 2017;23:703-13.

6. Tyran M, Carbuccia N, Garnier S, et al. A Comparison of DNA Mutation and Copy Number Profiles of Primary Breast Cancers and Paired Brain Metastases for Identifying Clinically Relevant Genetic Alterations in Brain 
Metastases. Cancers (Basel) 2019;11:665.

7. Gaedcke J, Traub F, Milde S, et al. Predominance of the basal type and HER-2/neu type in brain metastasis from breast cancer. Mod Pathol 2007;20:864-70.

8. Foulkes WD, Smith IE, Reis-Filho JS. Triple-negative breast cancer. N Engl J Med 2010;363:1938-48.

9. Mitri Z, Constantine T, O'Regan R. The HER2 Receptor in Breast Cancer: Pathophysiology, Clinical Use, and New Advances in Therapy. Chemother Res Pract 2012;2012:743193.

10. Zhang H, Berezov A, Wang Q, et al. ErbB receptors: from oncogenes to targeted cancer therapies. J Clin Invest 2007;117:2051-8.

11. Priedigkeit N, Hartmaier RJ, Chen Y, et al. Intrinsic Subtype Switching and Acquired ERBB2/HER2 Amplifications and Mutations in Breast Cancer Brain Metastases. JAMA Oncol 2017;3:666-71.

12. Cheng X, Hung MC. Breast cancer brain metastases. Cancer Metastasis Rev 2007;26:635-43.

13. Kabraji S, Ni J, Lin NU, et al. Drug Resistance in HER2Positive Breast Cancer Brain Metastases: Blame the Barrier or the Brain? Clin Cancer Res 2018;24:1795-804.

14. Shah N, Mohammad AS, Saralkar P, et al. Investigational chemotherapy and novel pharmacokinetic mechanisms for the treatment of breast cancer brain metastases. Pharmacol Res 2018;132:47-68.

15. Murthy RK, Loi S, Okines A, et al. Tucatinib, Trastuzumab, and Capecitabine for HER2-Positive Metastatic Breast Cancer. N Engl J Med 2020;382:597-609.

16. Mittapalli RK, Adkins CE, Bohn KA, et al. Quantitative Fluorescence Microscopy Measures Vascular Pore Size in Primary and Metastatic Brain Tumors. Cancer Res 2017;77:238-46.

17. Pegram MD. Neratinib in ERBB2-Positive Brain Metastases. JAMA Oncol 2016;2:1541-3.

18. Niwińska A, Murawska M, Pogoda K. Breast cancer brain metastases: differences in survival depending on biological subtype, RPA RTOG prognostic class and systemic treatment after whole-brain radiotherapy (WBRT). Ann Oncol 2010;21:942-8.

19. Liu JC, Zacksenhouse M, Eisen A, et al. Identification of cell proliferation, immune response and cell migration as critical pathways in a prognostic signature for HER2+:ER $\alpha$ - breast cancer. PLoS One 2017;12:e0179223.

20. Li CI, Zhang Y, Cieślik M, et al. Cancer Cell Intrinsic and Immunologic Phenotypes Determine Clinical Outcomes in Basal-like Breast Cancer. Clin Cancer Res 2021;27:3079-93.
21. Callens C, Driouch K, Boulai A, et al. Molecular features of untreated breast cancer and initial metastatic event inform clinical decision-making and predict outcome: long-term results of ESOPE, a single-arm prospective multicenter study. Genome Med 2021;13:44.

22. Bos PD, Zhang XH, Nadal C, et al. Genes that mediate breast cancer metastasis to the brain. Nature 2009;459:1005-9.

23. Davis S, Meltzer PS. GEOquery: a bridge between the Gene Expression Omnibus (GEO) and BioConductor. Bioinformatics 2007;23:1846-7.

24. Love MI, Huber W, Anders S. Moderated estimation of fold change and dispersion for RNA-seq data with DESeq2. Genome Biol 2014;15:550.

25. Wu T, Hu E, Xu S, et al. clusterProfiler 4.0: A universal enrichment tool for interpreting omics data. Innovation (N Y) 2021;2:100141.

26. Wickham H (2016). ggplot2: Elegant Graphics for Data Analysis.Springer-Verlag New York. ISBN 978-3-31924277-4. Available online: https://ggplot2.tidyverse.org.

27. Kassambara A, Kosinski M, Biecek P, et al. survminer: Drawing Survival Curves using 'ggplot2'. Version 202103-09. Available online: https://github.com/kassambara/ survminer

28. Lippitz BE. Cytokine patterns in patients with cancer: a systematic review. Lancet Oncol 2013;14:e218-28.

29. Coffelt SB, Kersten K, Doornebal CW, et al. IL-17producing $\gamma \delta \mathrm{T}$ cells and neutrophils conspire to promote breast cancer metastasis. Nature 2015;522:345-8.

30. Holst F, Stahl PR, Ruiz C, et al. Estrogen receptor alpha (ESR1) gene amplification is frequent in breast cancer. Nat Genet 2007;39:655-60.

31. Schmidt M, Böhm D, von Törne C, et al. The humoral immune system has a key prognostic impact in nodenegative breast cancer. Cancer Res 2008;68:5405-13.

32. Sun L, Chu H, Li H, Liu Y. LncRNA SNHG1 correlates with higher $\mathrm{T}$ stage and worse overall survival, and promotes cell proliferation while reduces cell apoptosis in breast cancer. Transl Cancer Res 2019;8:603-13.

33. Jin T, Xu X, Hereld D. Chemotaxis, chemokine receptors and human disease. Cytokine 2008;44:1-8.

34. Azimi I, Roberts-Thomson SJ, Monteith GR. Calcium influx pathways in breast cancer: opportunities for pharmacological intervention. Br J Pharmacol 2014;171:945-60.

35. Dustin D, Gu G, Fuqua SAW. ESR1 mutations in breast cancer. Cancer 2019;125:3714-28.

36. Razavi P, Dickler MN, Shah PD, et al. Alterations in 
PTEN and ESR1 promote clinical resistance to alpelisib plus aromatase inhibitors. Nat Cancer 2020;1:382-93.

37. Arnesen S, Blanchard Z, Williams MM, et al. Estrogen Receptor Alpha Mutations in Breast Cancer Cells Cause Gene Expression Changes through Constant Activity and Secondary Effects. Cancer Res 2021;81:539-51.

38. Huang RSP, Haberberger J, McGregor K, et al. Clinicopathologic and Genomic Landscape of Breast Carcinoma Brain Metastases. Oncologist 2021;26:835-44.

39. Stelzer G, Rosen N, Plaschkes I, et al. The GeneCards Suite: From Gene Data Mining to Disease Genome Sequence Analyses. Curr Protoc Bioinformatics 2016;54:1.30.1-1.30.33.

40. Komatsu M, Yoshimaru T, Matsuo T, et al. Molecular features of triple negative breast cancer cells by genome- wide gene expression profiling analysis. Int J Oncol 2013;42:478-506.

41. Edgar R, Domrachev M, Lash AE. Gene Expression Omnibus: NCBI gene expression and hybridization array data repository. Nucleic Acids Res 2002;30:207-10.

42. Gebremeskel S, Clattenburg DR, Slauenwhite D, et al. Natural killer $T$ cell activation overcomes immunosuppression to enhance clearance of postsurgical breast cancer metastasis in mice. Oncoimmunology 2015;4:e995562.

43. Lonser RR, Song DK, Klapper J, et al. Surgical management of melanoma brain metastases in patients treated with immunotherapy. J Neurosurg 2011;115:30-6.

(English Language Editor: A. Kassem)
Cite this article as: Wang P, Sun Z, Zhang Z, Yin Q. Immune response pathways enriched in breast cancer samples with brain metastasis. Gland Surg 2021;10(12):3334-3341. doi: 10.21037/gs$21-745$ 